\title{
Development of an experiment-based robust design paradigm for multiple quality characteristics using physical programming
}

\author{
Jami Kovach • Byung Rae Cho • Jiju Antony
}

Received: 18 April 2006 / Accepted: 5 September 2006 / Published online: 8 November 2006

(C) Springer-Verlag London Limited 2006

\begin{abstract}
The well-known quality improvement methodology, robust design, is a powerful and cost-effective technique for building quality into the design of products and processes. Although several approaches to robust design have been proposed in the literature, little attention has been given to the development of a flexible robust design model. Specifically, flexibility is needed in order to consider multiple quality characteristics simultaneously, just as customers do when judging products, and to capture design preferences with a reasonable degree of accuracy. Physical programming, a relatively new optimization technique, is an effective tool that can be used to transform design preferences into specific weighted objectives. In this paper, we extend the basic concept of physical programming to robust design by establishing the links of experimental design and response surface methodology to address designers' preferences in a multiresponse robust design paradigm. A numerical example is used to show the proposed procedure and the results obtained are validated through a sensitivity study.
\end{abstract}

\section{J. Kovach}

Department of Information and Logistics Technology,

312 Technology Building, University of Houston,

Houston, TX 77204, USA

B. R. Cho $(\square)$

Advanced Quality Engineering Laboratory,

Department of Industrial Engineering, 110 Freeman Hall,

Clemson University,

Clemson, SC 29634, USA

e-mail: bcho@ces.clemson.edu

\section{J. Antony}

Division of Management, Caledonian Business School,

Glasgow Caledonian University,

Cowcaddens Road,

Glasgow G4 0BA, UK
Keywords Multiresponse robust design - Physical programming · Optimization · Flexibility · Preferences . Experimental design $\cdot$ Response surface methodology

\section{Introduction}

1.1 The design process

A designer's main task is to apply scientific knowledge to the solution of technical problems and then to optimize that solution within the given constraints in order to determine the major attributes of the product, such as capability to meet product specifications, quality, and cost. During the early stages of the design process, there are many uncertainties involved in the decision-making process. As a design evolves, more information becomes available, and the designer's understanding of the situation grows. Figure 1 shows the relationship between the design timeline and knowledge about the design, where the solid lines indicate the current measurements regarding the design process. We can observe that knowledge and freedom have an inverse relationship over the course of the design timeline. This relationship is ironic in that the designer has maximum design freedom when the knowledge about the design is minimal, but in the later stages when there is more information available, the designer has very little design freedom. Ideally, the designer should have maximum freedom in the later stages of the design process, when more information concerning the design parameters is available. The dashed lines in Fig. 1 depict the potential time savings that could be achieved by improving design flexibility in the early stages of design. By providing flexibility in design, rework, time to market, and product costs could be reduced. Ways to achieve design flexibility 


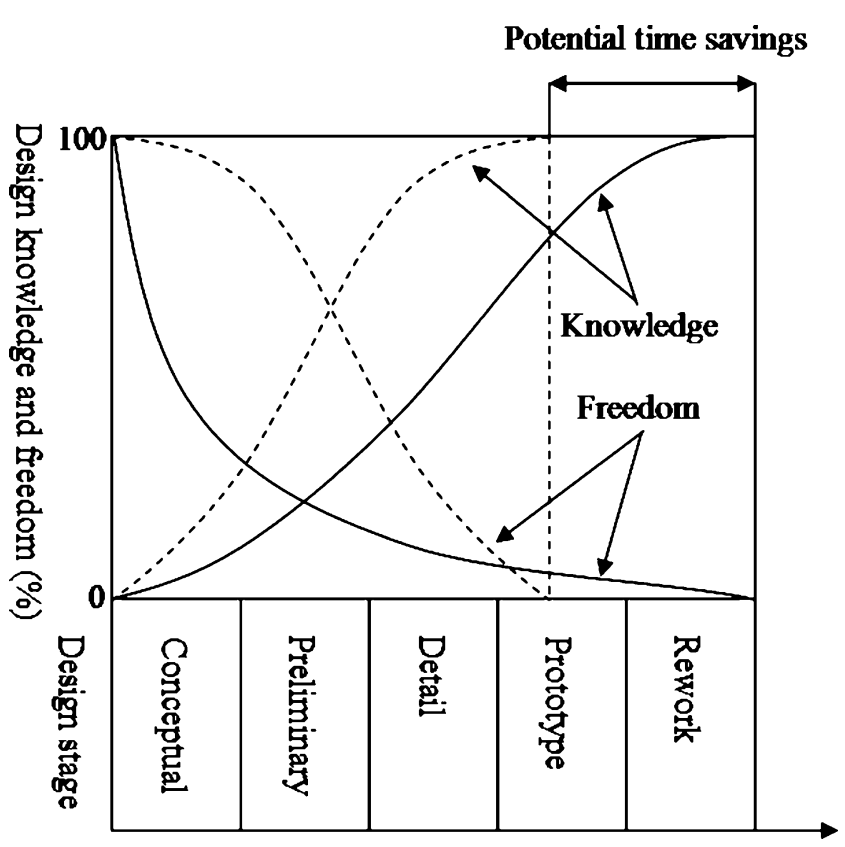

\section{Design timeline}

Fig. 1 Knowledge and freedom in the design process

include designing to accommodate changes or designing to anticipating changes that might occur. The latter method has proven unsuccessful because uncertainty is involved; therefore, the better solution is to design to accommodate changes with minimal rework cost.

\subsection{Literature review}

Among the design methods currently studied in engineering, researchers often identify robust design (RD) as one of the most important fields for the purpose of quality improvement. RD, which was first conceptualized by Taguchi $[1,2]$, is a cost-effective method to determine the optimum operating conditions of a system, using optimization techniques and design of experiments, in order to reduce costs and improve quality. By optimizing the design of products and processes, RD produces highquality products with low development time and manufacturing costs. A comprehensive analysis of RD can be found in $[3,4]$.

Taguchi's greatest contribution to the area of quality engineering was his quality philosophy, which simultaneously incorporates the mean and variability of a quality measure into product and process design in order to reduce variation. While the basic concept of RD is clearly important, Taguchi's assumptions, experimental design, and statistical analysis, have drawn much criticism. Complete discussions of the merits and deficiencies of Taguchi's
$\mathrm{RD}$ can be found in [5-12]. As a result, there have been many attempts in the literature to integrate Taguchi's RD principles with established statistical techniques, such as response surface methodology (RSM). RSM is a statistical tool that is useful for modeling and analysis in situations where the response of interest is controlled by several input factors. A comprehensive review of RSM is presented in [13].

Early work in this area by Vining and Myers [14] combines Taguchi's RD principles with conventional RSM in order to model the response of interest directly as a function of the design factors. This approach is known as the dual response model, where the goal is to minimize the process variance while adjusting the process mean to the desired target value. Further, work by del Castillo and Montgomery [15] and Copeland and Nelson [16] showed that standard nonlinear programming techniques, such as the generalized reduced gradient method and the NelderMead simplex method, may provide more effective RD solutions. It is important to note, however, that the dual response approach does not always guarantee optimal settings of design variables, which are often referred to as design factors, since the approach strictly requires a zerobias assumption by forcing the process mean to be located at the target value. To address this issue, Cho [17] and Lin and $\mathrm{Tu}$ [18] proposed the mean-squared error (MSE) approach by relaxing this zero-bias assumption. This approach shows that, while allowing some process bias, the resulting process variance would be less than or, at most, equal to the variance of the dual response approach; hence, the MSE approach provides better (or at least equal) settings of design factors than previous approaches. Further research in this area has been discussed in the literature [19-24].

Most traditional RD problems, such as those discussed previously, focus on a single-objective optimization approach, which considers only one performance measure. Several attempts have been made in the literature to optimize multiresponse problems in the context of RD. Four well-known methods are: (1) the contour overlay method, which uses visual inspection of superimposed response contour plots, but becomes less practical as the number of design variables increases; (2) the desirability function method (see $[25,26]$ ), which transforms the multiresponse problem into a single-response problem by maximizing the combined desirability; (3) the Khuri and Conlon [27] method, which uses the generalized distance approach to find the optimal settings that minimize the distance function over the experimental region; and (4) the loss function approach (see [28]), which uses a quadratic loss function to solve problems with multiple quality characteristics. Several other attempts have been made to optimize multiresponse problems; however, most of these 
methods are complex and inflexible. Although the RD methods reviewed here are clearly effective, there is room for improvement.

\subsection{Research motivation}

Most RD models reported in the literature consider a single quality characteristic. However, in most real-world industrial settings, multiple quality characteristics are often considered because customers judge products simultaneously on a variety of scales. Therefore, the objective should be to find the optimal settings for the design variables while considering multiple quality characteristics simultaneously. Yet, a vast majority of RD models fail to consider the implementation of design flexibility by not capturing preferences with a reasonable degree of accuracy. Therefore, most of these RD models involve iterative weight tweaking, as there is no clear method of prioritization. A physical programming approach to $\mathrm{RD}$ has been studied in [29-33]. The main focus of these papers was to improve design flexibility based on the preferences of the designer with the consideration of multiple quality characteristics using physical programming. As a further step, we propose a physical programming model for experimentbased RD by integrating the logistics of physical programming and experimental design by linking the concept of RSM to a multiresponse RD problem, which has not been fully studied in previous work. The proposed RD model is demonstrated through the use of a numerical example. A sensitivity analysis is also conducted in order to evaluate the effects of various model parameters on the results, which provides practitioners with insights into the practical implementation of the proposed model.

\section{Robust design considering multiple objectives}

Due to the practicality of multiresponse $\mathrm{RD}$, other work in this area is discussed here for the benefit of the reader. Logothetis and Haigh [34] used multiple regression and linear programming in a two-step approach to address multiresponse problems. Similar to Pignatiello's approach [28], Elsayed and Chen [35] and Ames et al. [36] also developed a multiple quality characteristic model based on the expected loss function. Derringer [37] further improved his approach to multiresponse problems by proposing a weighted desirability function. Kapur and Cho [38] proposed a multiresponse technique based on the criteria of minimizing deviation from the target and maximizing the robustness to noise, where a weighted penalty is incurred if the product characteristics deviate from their target values. An approach based on principal component analysis (PCA) was developed by $\mathrm{Su}$ and Tong [39]. Additionally, Tong and Su [40] utilized fuzzy set theory in weight-setting as an approach to optimize the multiresponse problem. Tong et al. [41] standardized the loss of each quality characteristic of interest so that standardization values ranged between 0 and 1. Chen [42] developed a multiple-quality-characteristic-based model where the designer's degree of satisfaction with each quality characteristic is represented by a transformation of the signal-to-noise ratio to a commensurable value between 0 and 1 . Kim and Lin [43] suggested a mathematical programming formulation for the dual response problem based on fuzzy optimization, called the fuzzy modeling approach. The goal is to identify the operating conditions that maximize the minimum degree of satisfaction with respect to the mean and variance within the feasible region. Vining [44] proposed a compromise approach to the multiresponse problem where the analyst can consider process economics and correlation structure. Tsui [45] used the expected loss function derived by Pignatiello [28] and extended this work to create a new model for other types of quality characteristics. Chen et al. [32] and Messac and Ismail-Yahaya [33] utilized physical programming to address the incorporation of designers' preferences into the multiresponse RD problem. Chiao and Hamada [46] proposed a multiple polynomial regression model to optimize the results of experiments where quality characteristics are correlated. To address the consideration of asymmetric quality loss, Kim and Cho [47] developed a priority-based RD model utilizing both the concepts of the dual response approach and nonlinear goal programming techniques. Based on the same goal programming techniques, Tang and $\mathrm{Xu}$ [48] proposed a unified formulation for the dual response optimization model by assigning different weights to bias and variability. Lin et al. [49] used fuzzy logic and Lu and Antony [50] used fuzzy-rule-based inference as separate approaches to the multiresponse problem. Romano et al. [51] utilized a total loss criterion, which raises customer satisfaction to the same level of concern as production costs. Wu and Chyu [52] built on Chiao and Hamada's model [46] by considering the loss coefficient of single characteristics and those between two correlated characteristics. The RD model proposed in this paper attempts to build on previous work in this area to further explore the simultaneous use of physical and goal programming approaches in multiresponse RD problems to better address process design and modeling flexibility issues by establishing links of experimental design to RD.

\section{Proposed approach}

In most cases, the designer has substantial knowledge about the problem at hand; however, in existing RD models, this knowledge is not utilized. In the proposed model, the 
knowledge possessed by the designer is exploited in order to impart design flexibility into the RD model for a multiresponse problem. The proposed model consists of the following steps:

1. Identify the design variables and quality characteristics of interest. This is often accomplished by screening experiments or is based on prior knowledge concerning the system under investigation.

2. Design an experiment to examine the chosen system variables and responses. Choose an experimental design that supports the desired analysis.

3. Conduct the experiment. Collect the specified data for each experimental trial.

4. Analyze the data. Estimate the fitted functions for the mean and variance of each quality characteristic using RSM.

5. Classify the mean and variance of each quality characteristic. Categorize the attributes based on the desired behavior of each response.

6. Incorporate preferences. Obtain preferences with respect to each quality attribute as specified by the designer and use these preferences to generate weights for use in the optimization model.

7. Build the objective function. Use preference weights and deviation variables to form an aggregate objective function.

8. Determine the optimal process parameter settings. Use the optimization model developed here to obtain the optimum operating conditions.

The planning through analysis stages of the proposed model follows the traditional RD method closely, in terms of experimental design and response modeling. However, during experimentation, data are collected for multiple responses, and fitted functions are determined for the mean and variance of each quality characteristic of interest. These equations are then used simultaneously to solve the multiresponse problem using the classification method and optimization model proposed in this work. These techniques are further developed in the following sections.

\subsection{Method of classification}

The proposed RD model uses a physical programming framework, but the framework has been customized to fit the particular model under consideration through the incorporation of goal programming techniques. In the proposed model, the estimated response surface functions for the process mean and variance are treated as individual quality attributes. Then, trade-offs between quality characteristics are made by allowing the designer to express preferences in a fashion that is less rigid and more flexible than traditional methods. Using the physical programming approach, the preferences expressed by the designer are classified into one of four classes, and each class includes two sub-cases (hard and soft cases). Thus, the desired behavior of the mean and variance of each quality characteristic is classified into one of these categories, which are shown in Table 1. Graphical representations of the qualitative meaning of each class are displayed in Table 2 .

A quality characteristic is classified as a soft class if it has a preferred target value where it needs to be either minimized, maximized, or should fall within a desired range. But, as the name suggests, these requirements are flexible. The mean function of a quality characteristic can be classified into any one of the four classes; however, the standard deviation always belongs to class $1 \mathrm{~S}$ due to the fact that, in $\mathrm{RD}$, the variance is preferred to be as small as possible. Oppositely, if the preferred value of a quality attribute is not flexible in that it is either feasible or infeasible, then it is classified as a hard class. The hard classes are applicable in situations where the designer must stay within a specified range. The values associated with hard classes are either acceptable or not acceptable; there are no other distinctions in the hard classes. For each response, the designer is asked to express their preferences in detail by specifying different ranges of acceptability. These ranges are based on the designer's prior knowledge concerning the system under investigation and/or historical data. Then, based on these ranges, a class function is formed which determines how sensitive the solution would be to the design specifications. These functions map the units and physical meaning of each response range to a

Table 1 Definitions of quality characteristic classifications

\begin{tabular}{|c|c|c|c|}
\hline Case & Class & Classification & Interpretation \\
\hline \multirow[t]{4}{*}{ Soft } & $1 \mathrm{~S}$ & Smaller-the-better & Minimize $\widehat{\mu}_{i}(\mathbf{x})$ or $\widehat{\sigma}_{i}(\mathbf{x})$ \\
\hline & $2 \mathrm{~S}$ & Larger-the-better & Maximize $\widehat{\mu}_{i}(\mathbf{x})$ \\
\hline & $3 \mathrm{~S}$ & Nominal-the-better & $\begin{array}{l}\text { A specified target value for } \\
\widehat{\mu}_{i}(\mathbf{x}) \text { is preferred }\end{array}$ \\
\hline & $4 \mathrm{~S}$ & Desired range & $\begin{array}{l}\hat{\mu}_{i}(\mathbf{x}) \text { is preferred within a } \\
\text { particular range of values }\end{array}$ \\
\hline \multirow[t]{4}{*}{ Hard } & $1 \mathrm{H}$ & Less than & $\begin{array}{l}\widehat{\mu}_{i}(\mathbf{x}) \leq \mu_{i, \max } \text { or } \widehat{\sigma}_{i}(\mathbf{x}) \leq \\
\sigma_{i, \max }, \text { where } \mu_{i, \max } \text { and } \\
\sigma_{i, \max } \text { are assumed to be the } \\
\text { largest allowable values }\end{array}$ \\
\hline & $2 \mathrm{H}$ & Greater than & $\begin{array}{l}\widehat{\mu}_{i}(\mathbf{x}) \mu_{i, \min }, \text { where } \mu_{i, \min } \text { is } \\
\text { the smallest allowable value }\end{array}$ \\
\hline & $3 \mathrm{H}$ & Equal to & $\begin{array}{l}\widehat{\mu}_{i}(\mathbf{x})=\mu_{i, \text { nom }}, \text { where } \mu_{i, \text { nom }} \\
\text { is the desired target value }\end{array}$ \\
\hline & $4 \mathrm{H}$ & Required range & $\begin{array}{l}\mu_{i, \min } \leq \widehat{\mu}_{i}(\mathbf{x}) \leq \mu_{i, \max } \\
\text { where } \mu_{i, \max } \text { and } \mu_{i, \min } \text { are } \\
\text { the largest and smallest } \\
\text { allowable values, } \\
\text { respectively }\end{array}$ \\
\hline
\end{tabular}


Table 2 Graphical representations of quality characteristic classifications (adapted from [30])

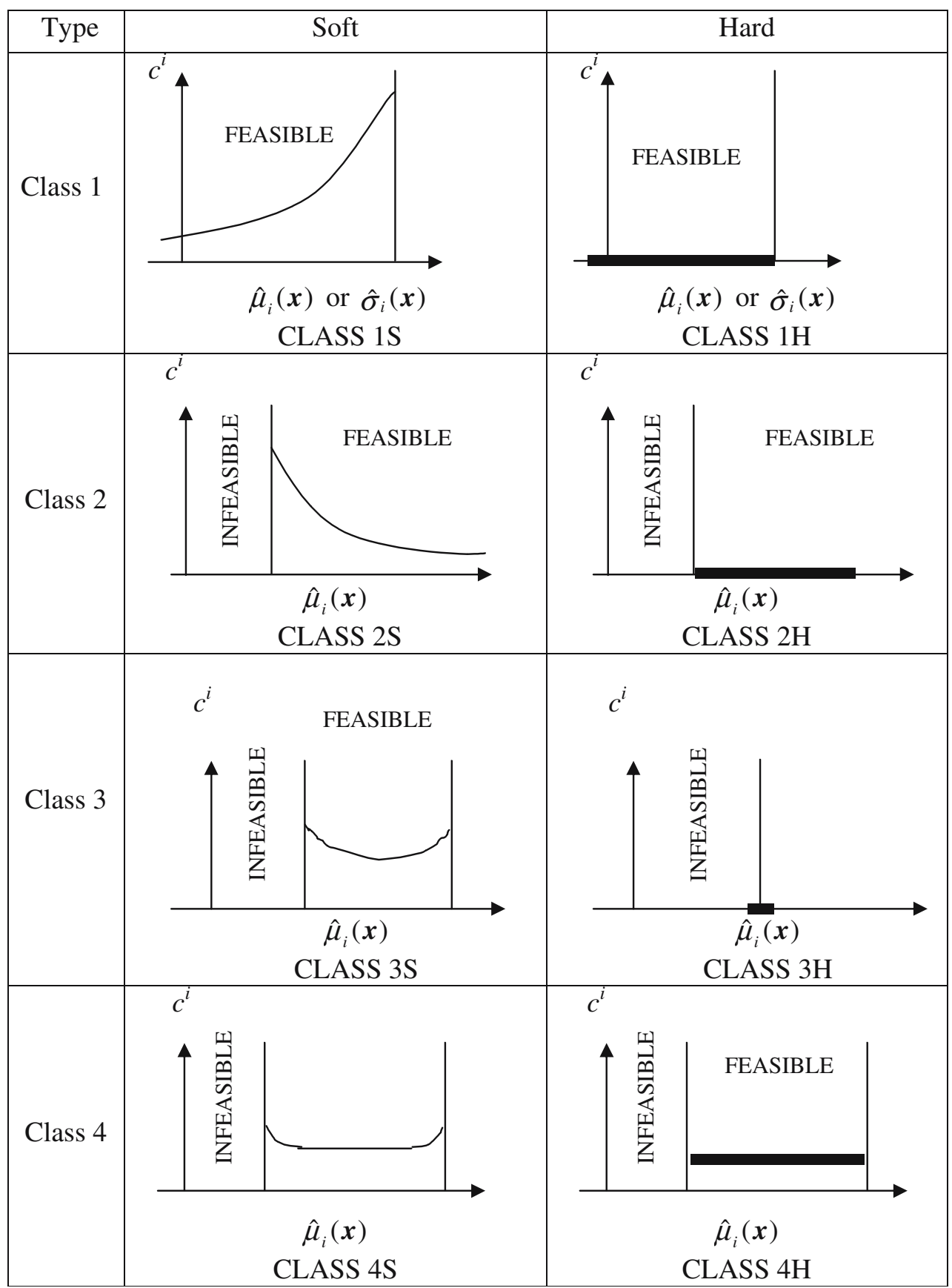

dimensionless scale, which is represented as a unimodal function and guides the path of the optimization process.

After classifying each of the quality characteristics into one of the four classes, the designer has to specify preferred values based on the extent of preference for each class. For each quality characteristic belonging to classes $1 \mathrm{~S}$ and $2 \mathrm{~S}$, the designer has to specify six ranges of preferred values, while for classes $3 \mathrm{~S}$ and $4 \mathrm{~S}$, ten and eleven ranges are specified, respectively. The six ranges for classes $1 \mathrm{~S}$ and $2 \mathrm{~S}$ are defined in Table 3. Figure 2 shows the class function for the standard deviation of a quality characteristic. Here, we use the notation where $\mathbf{x}$ is the set of design variables and the fitted functions of the mean and standard deviation are $\widehat{\mu}_{i}(\mathbf{x})$ and $\widehat{\sigma}_{i}(\mathbf{x})$, respectively. The value of the criterion under consideration, $\widehat{\sigma}_{i}(\mathbf{x})$, lies along the horizontal axis, and the class function, $c^{i}$, is depicted on the vertical axis. Similar diagrams can be created to examine the criterion $\widehat{\mu}_{i}(\mathbf{x})$ and/or other classes (see [30]); however, the shape of the class function depends on the numerical values of the range limits for the preferences specified by the designer. 
Table 3 Interpretation of ranges for classes $1 \mathrm{~S}$ and $2 \mathrm{~S}$

\begin{tabular}{|c|c|c|}
\hline Range & Notation $(s)$ & Interpretation \\
\hline Ideal & 1 & $\begin{array}{l}\text { A range in which every value is } \\
\text { considered to be ideal from the } \\
\text { designer's perspective. It is } \\
\text { important to note that any two points } \\
\text { inside this range are considered to } \\
\text { have equal values. }\end{array}$ \\
\hline Desirable & 2 & An acceptable range that is desirable. \\
\hline Tolerable & 3 & An acceptable range that is tolerable. \\
\hline Undesirable & 4 & $\begin{array}{l}\text { An acceptable range that is } \\
\text { undesirable. }\end{array}$ \\
\hline $\begin{array}{l}\text { Highly } \\
\text { undesirable }\end{array}$ & 5 & $\begin{array}{l}\text { An acceptable range that is highly } \\
\text { undesirable. }\end{array}$ \\
\hline Unacceptable & $\mathrm{n} / \mathrm{a}$ & $\begin{array}{l}\text { An unacceptable range of values that } \\
\text { is not permissible. }\end{array}$ \\
\hline
\end{tabular}

\subsection{Proposed optimization model}

During the development of the optimization model, fitted functions for the different quality characteristics of interest are estimated using RSM in order to find the optimum operating conditions. It is often the case, however, that different characteristics have conflicting objectives. For example, one characteristic has to be minimized and another has to be maximized. Furthermore, if there are many characteristics involved, it becomes very difficult to reach a compromise between these conflicts in order to obtain robust operating parameters. In the proposed RD model, an aggregate objective function (AOF) is formed, which is a representation of all of the individual quality characteristics and the designer's preferences. Once the AOF is formed, the optimization model resembles a conventional optimization model, which can determine the optimal solution with respect to all of the responses. The method of forming the AOF is the main differentiating aspect of our proposed model versus conventional models. Here, we use the desirability ranges specified by the designer and their respective class functions to determine importance weightings for each quality characteristic categorized as a soft case. These weights are then multiplied by the deviation from the desired value with respect to each range, which is summed to create the AOF.

The AOF embodies the full complexity of the designer's preferences using physical programming techniques. All of the soft class functions are combined together to form the $\mathrm{AOF}$ and the hard classes become the constraints in the optimization model. The class function is the function that is minimized for the quality attribute under consideration. A lower value of a class function is preferred over a higher value because the class function is convex and the lower value corresponds to the optimal value of the quality attribute. From Fig. 2, we can see that the value of the class function at the ideal value of the desired quality attribute is zero. For analysis of other categories of quality characteristics, see [29-31]. The AOF will be a true reflection of the designer's preferences only if it takes into consideration both inter-criteria and intra-criteria preferences. The intercriteria preferences are captured by enforcing a one versus others (OVO) criteria rule. If a situation arises during the optimization process, there are two choices available: (1) one quality attribute can improve from the undesirable range to the tolerable range and (2) all of the other quality attributes can improve from the tolerable to desirable range. The OVO property of the class function ensures that the first option is always selected in situations where only one of the two improvements can be made.

In physical programming, a set of weights that represents the designer's preference is calculated for all quality attributes based on the ranges of different degrees of desirability for each attribute determined by the designer. The final set of weights for a particular interval is given by the difference in slope for adjoining intervals in the class function. Thus, in order to calculate the final set of weights, we need to compute the slopes of the different intervals in the class function. First, in order to calculate the slope, we need to determine the rate of change along the horizontal axis with respect to the rate of change along the vertical axis. The rate of change along the horizontal axis is given by the following relationships:

Case 1 (consideration of the mean of the $i^{\text {th }}$ quality characteristic):

$$
\begin{aligned}
& \widehat{\mu}_{i s R}=\mu_{i s R}-\mu_{i(s-1) R}, \text { for } 2 \leq s \leq 5 \\
& \widehat{\mu}_{i s L}=\mu_{i s L}-\mu_{i(s-1) L}, \text { for } 2 \leq s \leq 5
\end{aligned}
$$

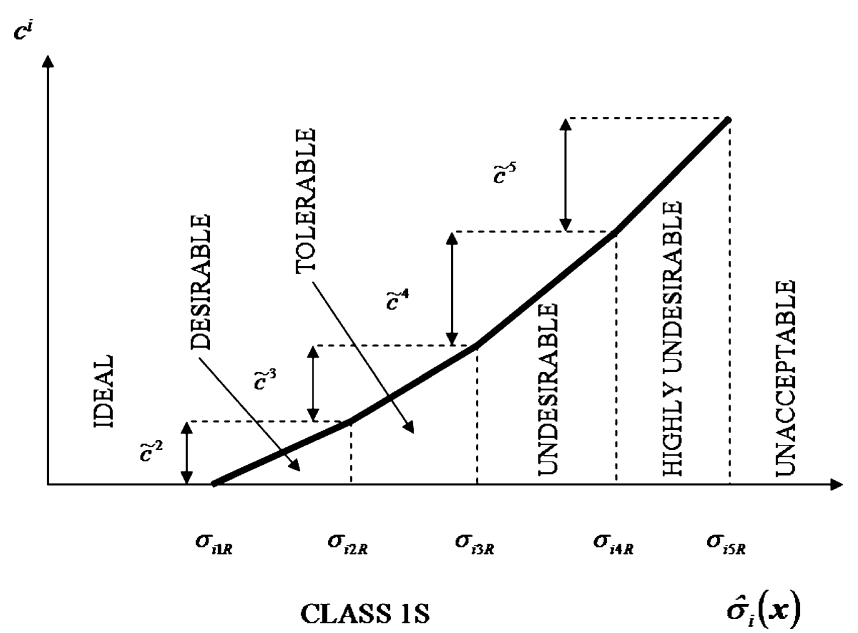

Fig. 2 Class function for the standard deviation of the $i^{\text {th }}$ quality characteristic (adapted from [30]) 
Case 2 (consideration of the standard deviation of the $i^{\text {th }}$ quality characteristic):

$$
\widehat{\sigma}_{i s R}=\sigma_{i s R}-\sigma_{i(s-1) R}, \text { for } 2 \leq s \leq 5
$$

Note that $\mu_{i 1 R}$ to $\mu_{i s R}, \mu_{i 1 L}$ to $\mu_{i s L}$, and $\sigma_{i 1 R}$ to $\sigma_{i s R}$ are physically meaningful values of quality attributes specified by the designer, where $R$ and $L$ represent the right and left sides of the class function, respectively, and $s$ describes the range/range limit. For example, $\widehat{\mu}_{i s R}$ is the estimated mean of the $i^{\text {th }}$ quality characteristic in the $s$ th range on the right side of the class function, and $\mu_{i s}$ is the mean of the $i$ th quality characteristic for the lower limit of the $s^{\text {th }}$ range on the right side of the class function; hence, $1 \leq S \leq 5$, since only five limits are needed to specify six ranges (see Fig. 2). The next step is to determine the rate of change along the vertical axis. The rate of change that takes place as we travel along the $s^{\text {th }}$ range is given by:

$\widetilde{c}^{s}=c^{s}-c^{s-1}$, for $2 \leq s \leq 5$

where $\widetilde{c}^{s}$ is the rate of change in $c^{i}$ that takes place as we travel along the $s^{\text {th }}$ range on the horizontal axis. In the above relation, we do not know the values of $c^{i}$ to calculate the values of $\widetilde{c}^{s}$. Using the knowledge about the properties of the class functions, we can express the value that $\widetilde{c}^{s}$ should possess. By defining the values of $\widetilde{c}^{s}$, we can enforce the OVO rule and the convexity requirement. That is:

$\widetilde{c}^{s}=\gamma\left(n_{\mathrm{sc}}-1\right) \widetilde{c}^{s-1}$, for $2 \leq s \leq 5, n_{\mathrm{sc}}>1$, and $\gamma>1$

where $\gamma$ is the convexity parameter introduced to enforce the convexity property of the class function and $n_{\mathrm{sc}}$ is the number of soft criteria. If the class function is not found to be convex, then the value of $\gamma$ is increased until the convexity requirement is satisfied. From practice, the initial value of $\gamma$ usually considered is 1.1. The OVO rule is enforced by multiplying $\left(n_{\mathrm{sc}}-1\right)$ by the value of $\widetilde{c}^{s-1}$. This ensures that the penalty for an attribute staying in the tolerable range is significantly worse than staying in the desirable range. In order to apply the equation, we need the value of $\widetilde{c}^{2}$. This value is assumed to be a small positive number, such as 0.1 , since, logically, the rate of change along the first range of the vertical axis will be small. The next step is to calculate the slopes for the $s$ ranges. The slopes are calculated using the following relationships:

Case 1 (consideration of the mean of the $i^{\text {th }}$ quality characteristic):

$$
\begin{gathered}
w_{i s R}=\frac{\widetilde{c}^{s}}{\widehat{\mu}_{i s R}}, \text { for } 2 \leq s \leq 5 \\
w_{i s L}=\frac{\widetilde{c}^{s}}{\widehat{\mu}_{i s L}}, \text { for } 2 \leq s \leq 5
\end{gathered}
$$

Case 2 (consideration of the standard deviation of the $i^{\text {th }}$ quality characteristic):

$w_{i s R}=\frac{\widetilde{c}^{s}}{\widehat{\sigma}_{i s R}}$, for $2 \leq s \leq 5$

where $w_{i s L}$ and $w_{i s R}$ are the slopes for the different ranges.

Finally, the weights to be used in constructing the AOF are calculated by the following relationships:

Case 1 (consideration of the mean of the $i^{\text {th }}$ quality characteristic):

$$
\begin{gathered}
\widetilde{w}_{i s R}=w_{i s R}-w_{i(s-1) R}, \text { for } 2 \leq s \leq 5 \\
\widetilde{w}_{i s L}=w_{i s L}-w_{i(s-1) L}, \text { for } 2 \leq s \leq 5 \\
w_{i 1 L}=w_{i 1 R}=0
\end{gathered}
$$

Case 2 (consideration of the standard deviation of the $i^{\text {th }}$ quality characteristic):

$\widetilde{w}_{i s R}=w_{i s R}-w_{i(s-1) R}$, for $2 \leq s \leq 5$

where $\widetilde{w}_{i s L}$ and $\widetilde{w}_{i s R}$ are the calculated weights. The calculated weights signify the penalty for deviating from the preferred value of a particular range. The penalty at the ideal range is zero because the target value is achieved and there is no deviation, so the values of $w_{i 1 L}=w_{i 1 R}$ are considered to be zero. The next step is to verify that the value assumed for the convexity parameter is adequate. This is done by using the following expression:

$$
\widetilde{w}_{\min }=\min _{1, s}\left\{\widetilde{w}_{i s R}, \widetilde{w}_{i s L}\right\}>0, \text { for } 2 \leq s \leq 5
$$

The difference in slope calculated along the ranges should be positive so that the convexity property of the class function is satisfied. This is verified using the relationship in Eq. 12. If the value of $\widetilde{w}_{\min }$ is smaller than a small chosen number, such as 0.01 , then the value of the convexity parameter is increased and the whole weight calculation process is repeated. Finally, the complete optimization model is given in Table 4. The purpose of this paper is not to discuss the mathematical details of physical programming. For these details, the reader is referred to [29-31].

The weights that are computed represent the penalties for the attributes if they deviate from the desired value of each 
Table 4 Robust design optimization model using physical programming

Mathematical model

$$
\begin{aligned}
& \text { Find: } \mathbf{x}^{*} \\
& \text { Minimize: AOF }=\sum_{i=1}^{n_{\mathrm{sc}}} \sum_{s=2}^{5}\left(\widetilde{w}_{i s L} d_{i s}^{-}+\widetilde{w}_{i s R} d_{i s}^{+}\right) \\
& \text {Satisfy: } \\
& \widehat{\mu}_{i}(\mathbf{x}) \leq \mu_{i, \max } \forall i \text { in class } 1 \mathrm{H}, i=1,2, \ldots, n_{\mathrm{hc}} \\
& \widehat{\sigma}_{i}(\mathbf{x}) \leq \sigma_{i, \max } \forall i \text { in class } 1 \mathrm{H}, i=1,2, \ldots, n_{\mathrm{hc}} \\
& \widehat{\mu}_{i}(\mathbf{x}) \geq \mu_{i, \min } \forall i \text { in class } 2 \mathrm{H}, i=1,2, \ldots, n_{\mathrm{hc}} \\
& \widehat{\mu}_{i}(\mathbf{x})=\mu_{i, \text { nom }} \forall i \text { in class } 3 \mathrm{H}, i=1,2, \ldots, n_{\mathrm{hc}} \\
& \mu_{i, \text { min }} \leq \widehat{\mu}_{i}(\mathbf{x}) \leq \mu_{i, \text { max }} \forall i \text { in class } 4 \mathrm{H}, i=1,2, \ldots, n_{\mathrm{hc}} \\
& \mathbf{x}_{\mathrm{min}} \leq \mathbf{x} \leq \mathbf{x}_{\text {max }} \\
& \widehat{\mu}_{i}(\mathbf{x})=\mathbf{X} \widehat{\beta}_{i}, \text { where } \beta_{i}=\left(\mathbf{X}^{\mathrm{T}} \mathbf{X}\right)^{-1} \mathbf{X}^{\mathrm{T}} \bar{y}_{i} \\
& \widehat{\sigma}_{i}(\mathbf{x})=\mathbf{X} \widehat{\delta}_{i}, \text { where } \delta_{i}=\left(\mathbf{X}^{\mathrm{T}} \mathbf{X}\right)^{-1} \mathbf{X}^{\mathrm{T}} \sigma_{i} \\
& \widehat{\mu}_{i}(\mathbf{x})-d_{i s}^{+} \leq \mu_{i(s-1) R} ; d_{i s}^{+} \geq 0 ; \widehat{\mu}_{i}(\mathbf{x}) \leq \mu_{i 5 R} \\
& \quad \forall i \text { in classes } 1 \mathrm{~S}, 3 \mathrm{~S}, 4 \mathrm{~S}, i=1,2, \ldots, 5 \\
& \widehat{\sigma}_{i}(\mathbf{x})-d_{i s}^{+} \leq \sigma_{i(s-1) R} ; d_{i s}^{+} \geq 0 ; \widehat{\sigma}_{i}(\mathbf{x}) \leq \sigma_{i 5 R} \\
& \quad \forall i \text { in class } 1 \mathrm{~S}, i=1,2, \ldots, n_{\mathrm{sc}}, s=2, \ldots, 5 \\
& \widehat{\mu}_{i}(\mathbf{x})-d_{i s}^{-} \leq \mu_{i(s-1) L} ; d_{i s}^{-} \geq 0 ; \widehat{\mu}_{i}(\mathbf{x}) \leq \mu_{i 5 L} \\
& \quad \forall i \text { in classes } 1 \mathrm{~S}, 3 \mathrm{~S}, 4 \mathrm{~S}, i=1,2, \ldots, n_{\mathrm{sc}}, s=2, \ldots, 5 \\
& \text { where: } \\
& n_{\mathrm{hc}}=\text { number of hard criteria } \\
& n_{\mathrm{sc}}=\text { number of soft criteria } \\
& \mu_{i, \text { max }}=\text { maximum value of the response specified by the designer } \\
& \mu_{i, \text { min }}=\text { minimum value of the response specified by the designer } \\
& \mu_{i, \text { nom }}=\text { desired target value of the response specified by the designer } \\
& \mathbf{x}=\text { control factor vector } \\
& d_{i s}^{+}, d_{i s}^{-}=\text {deviational variables }
\end{aligned}
$$

range. During the optimization process, the deviation from the desired value is captured using the deviation variables $\left(d_{i s}^{-}, d_{i s}^{+}\right)$, which are penalized with respect to the deviation from each of the four ranges. For example, the penalty for deviating from the preferred value of the tolerable range is much higher than the penalty of deviating from the preferred value of the desirable range. The values of deviational variables are multiplied by the weights with respect to each range. This process is repeated for all of the soft classes and the values are summed to form the AOF. The set of process parameter values which results in the minimum value subject to the hard constraints for the computed AOF is chosen as the optimal process parameter settings. That is, the AOF is as follows:

$\operatorname{Minimize} \sum_{i} \sum_{s}\left(\widetilde{w}_{i s L} d_{i s}^{-}+\widetilde{w}_{i s R} d_{i s}^{+}\right)$

This minimization of the AOF is performed using customized optimization software called PhyOpt, which was developed using C\# (C sharp), a programming language from Microsoft.
3.3 Comparison of the proposed methodology to existing approaches

The methodology proposed in this paper follows a dimensionality reduction strategy, where a multiresponse problem is transformed into a single-response problem in order to make it easier to solve via conventional optimization methods. The use of this strategy is the usual approach to this type of problem and is illustrated by other multiresponse solutions methods, such as those discussed in Section 1.2. Through the use of goal programming techniques (see [53]), the AOF is formed by way of deviation variables, $\left(d_{i s}^{-}, d_{i s}^{+}\right)$, mapped to each desired response for the soft classes and the hard classes become the model constraints. Yet, the approach proposed in this work is unique because it also incorporates a priority-based strategy in that each deviation variable is given a priority or a weight. This model extends the work proposed by Chen et al. [32], Messac and Ismail-Yahaya [33], and Kim and Cho [47] through the use of physical programming to determine the weights used in the optimization model, which is combined with the use of traditional experimental design and modeling techniques. This approach creates a clearly defined method of prioritization for use in multiresponse problems; however, because this method is ultimately based on designer preferences, which are hard to determine specifically, there is still a degree of uncertainty associated with this model. Therefore, validity and robustness can not be guaranteed. Yet, the proposed methodology is an improvement over current approaches because it provides a larger degree of flexibility than previous approaches, due to the independent nature of its model components. The flexibility of the proposed approach is highlighted in the numerical example illustrated in the following section.

\section{Numerical example}

Here, we investigate an example from Bourquin et al. [54] which considers a tablet manufacturing problem from the pharmaceutical industry. There are several design factors that can be manipulated to achieve a certain dosage parameter for each tablet. The goal here is to optimize the process parameter settings, which include silica aero gel, dwell time/compression speed, and compression force, in order to achieve the desired responses. The quality characteristics considered in this problem are: (1) tensile strength, (2) disintegration time, and (3) weight. A central composite design was used to generate the response data. The estimated response functions of the process mean and standard deviation for the three quality 
attributes considered in this example are found as follows:

$$
\begin{aligned}
& \widehat{\mu}_{1}(\mathbf{x})=175+12 x_{1}+15.3 x_{2}+29.2 x_{3}+4.2 x_{1}^{2}-1.3 x_{2}^{2}+16.8 x_{3}^{2}+7.7 x_{1} x_{2}+5.1 x_{1} x_{3}+14.1 x_{2} x_{3} \\
& \widehat{\sigma}_{1}(\mathbf{x})=25.2+2.8 x_{1}+1.06 x_{2}+0.55 x_{3}+2.94 x_{1}^{2}-8.14 x_{2}^{2}-2.72 x_{3}^{2}+6.71 x_{1} x_{2}+9.35 x_{1} x_{3}+2.15 x_{2} x_{3} \\
& \widehat{\mu}_{2}(\mathbf{x})=84.9+5.3 x_{1}+0.24 x_{2}+8.80 x_{3}-0.52 x_{1}^{2}-11.80 x_{2}^{2}+0.39 x_{3}^{2}+0.22 x_{1} x_{2}+3.60 x_{1} x_{3}-4.42 x_{2} x_{3} \\
& \widehat{\sigma}_{2}(\mathbf{x})=25.4+0.2 x_{1}+0.031 x_{2}+0.4 x_{3}-2.741 x_{1}^{2}-8.91 x_{2}^{2}-2.54 x_{3}^{2}+6.397 x_{1} x_{2}+9.243 x_{1} x_{3}+1.601 x_{2} x_{3} \\
& \widehat{\mu}_{3}(\mathbf{x})=39.7+3.6 x_{1}+1.002 x_{2}+2.26 x_{3}+2.542 x_{1}^{2}-9.69 x_{2}^{2}-2.37 x_{3}^{2}+6.083 x_{1} x_{2}+9.135 x_{1} x_{3}+1.051 x_{2} x_{3} \\
& \widehat{\sigma}_{3}(\mathbf{x})=33.3+1.8 x_{1}+0.0999 x_{2}+3.0 x_{3}+2.311 x_{1}^{2}-11.60 x_{2}^{2}-2.17 x_{3}^{2}+5.823 x_{1} x_{2}+9.398 x_{1} x_{3}+0.114 x_{2} x_{3}
\end{aligned}
$$

The attributes of each of the quality characteristics are then classified and the designer's preferences are specified. This information is shown in Table 5 and is based on the designer's judgment concerning the system under investigation. The next step is to assess the weights based on the preferences of the designer. For demonstration purposes, only the derivation of the weights for the mean of the tensile strength $\left(\widehat{\mu}_{1}(\mathbf{x})\right)$ is shown here. First, the rates of change along the horizontal axis are calculated using Eq. 2:

$\widehat{\mu}_{12 L}=175-171.25=3.75$

$\widehat{\mu}_{13 L}=171.25-167.5=3.75$

$\widehat{\mu}_{14 L}=167.5-163.75=3.75$

$\widehat{\mu}_{15 L}=163.75-160=3.75$

The rates of change along the vertical axis are determined using Eq. 5:

$\gamma=1.1$

$n_{\mathrm{sc}}=6$

$\widetilde{c}^{2}=0.1$

$\widetilde{c}^{3}=1.1 *(6-1) * 0.1=0.55$

$\widetilde{c}^{4}=1.1 *(6-1) * 0.55=3.025$

$\widetilde{c}^{5}=1.1 *(6-1) * 3.025=16.6375$

Then, using the results from the previous calculations, the slopes are computed using Eq. 7:

$$
\begin{aligned}
& w_{12 L}=\frac{0.1}{3.75}=0.0267 \\
& w_{13 L}=\frac{0.55}{3.75}=0.1466 \\
& w_{14 L}=\frac{3.025}{3.75}=0.8066 \\
& w_{15 L}=\frac{16.637}{3.75}=4.4366
\end{aligned}
$$

Finally, the weights are calculated using Eq. 10:

$$
\begin{aligned}
& \widetilde{w}_{12 L}=0.0267-0=0.0267 \\
& \widetilde{w}_{13 L}=0.1466-0.0267=0.1199 \\
& \widetilde{w}_{14 L}=0.8066-0.1466=0.66 \\
& \widetilde{w}_{15 L}=4.4366-0.8066=3.63
\end{aligned}
$$

This procedure is also used to determine the weights for the attributes of the other quality characteristics of interest in this example, all of which are then combined with the deviation variables and summed to form the AOF. Based on the model given in Table 4 and the AOF given in Eq. 13, the optimal process parameter settings found using PhyOpt are shown in Table 6.

\subsection{Discussion}

A summary of the process followed in this example is shown in Fig. 3, where the flow of the process is broken down into four major sections and each step of the process is outlined in detail. Here, we analyze the impact of design changes on the proposed model. During the design process, there is a potential for changes in the designer's preferences (i.e., changes in the ranges of different degrees of desirability for soft classes). Once the designer's preferences are re-specified, the original model can be adjusted by simply recalculating the weights associated with those quality characteristics whose preferences changed. These new weights are then easily combined with the weights previously calculated, for those quality characteristics whose preferences did not change, to create the AOF. The new optimum operating conditions are then determined by optimizing the revised AOF. Additionally, in designing new products and processes, the categorization of a quality characteristic of interest may change. These situations may arise in one of two ways. It may be necessary to change the 
Table 5 Preferences of the designer for the tablet manufacturing problem

\begin{tabular}{|c|c|c|c|c|}
\hline $\begin{array}{l}\text { Quality } \\
\text { characteristic }\end{array}$ & Class & Ranges & $\begin{array}{l}\text { Notation } \\
(s)\end{array}$ & Numerical values \\
\hline \multirow[t]{6}{*}{ Mean (1) } & \multirow[t]{6}{*}{$2 \mathrm{~S}$} & Ideal & 1 & $>175$ \\
\hline & & Desirable & 2 & 171.5 to 175 \\
\hline & & Tolerable & 3 & 167.5 to 171.25 \\
\hline & & Undesirable & 4 & 163.75 to 167.5 \\
\hline & & $\begin{array}{l}\text { Highly } \\
\text { undesirable }\end{array}$ & 5 & 160 to 163.75 \\
\hline & & Unacceptable & $\mathrm{n} / \mathrm{a}$ & $<160$ \\
\hline \multirow{6}{*}{$\begin{array}{l}\text { Standard } \\
\text { deviation (1) }\end{array}$} & \multirow[t]{6}{*}{$1 \mathrm{~S}$} & Ideal & 1 & $<0.5$ \\
\hline & & Desirable & 2 & 0.5 to 1.5 \\
\hline & & Tolerable & 3 & 1.5 to 2.0 \\
\hline & & Undesirable & 4 & 2.0 to 3.5 \\
\hline & & $\begin{array}{l}\text { Highly } \\
\text { undesirable }\end{array}$ & 5 & 3.5 to 6.0 \\
\hline & & Unacceptable & $\mathrm{n} / \mathrm{a}$ & $>6.0$ \\
\hline \multirow[t]{6}{*}{ Mean (2) } & \multirow[t]{6}{*}{$3 \mathrm{~S}$} & Ideal & 1 & 70 \\
\hline & & Desirable & 2 & $\begin{array}{l}69.25 \text { to } 70 \text { and } 70 \\
\text { to } 70.75\end{array}$ \\
\hline & & Tolerable & 3 & $\begin{array}{l}68.5 \text { to } 69.25 \text { and } \\
70.75 \text { to } 71.5\end{array}$ \\
\hline & & Undesirable & 4 & $\begin{array}{l}67.75 \text { to } 68.5 \text { and } \\
71.5 \text { to } 72.25\end{array}$ \\
\hline & & $\begin{array}{l}\text { Highly } \\
\text { undesirable }\end{array}$ & 5 & $\begin{array}{l}67 \text { to } 67.75 \text { and } \\
72.25 \text { to } 73\end{array}$ \\
\hline & & Unacceptable & $\mathrm{n} / \mathrm{a}$ & $<67$ and $>73.00$ \\
\hline \multirow{6}{*}{$\begin{array}{l}\text { Standard } \\
\text { deviation (2) }\end{array}$} & \multirow[t]{6}{*}{$1 \mathrm{~S}$} & Ideal & 1 & $<0.5$ \\
\hline & & Desirable & 2 & 0.5 to 1.5 \\
\hline & & Tolerable & 3 & 1.5 to 2.0 \\
\hline & & Undesirable & 4 & 2.0 to 3.5 \\
\hline & & $\begin{array}{l}\text { Highly } \\
\text { undesirable }\end{array}$ & 5 & 3.5 to 5.0 \\
\hline & & Unacceptable & $\mathrm{n} / \mathrm{a}$ & $>5.0$ \\
\hline \multirow[t]{6}{*}{ Mean (3) } & \multirow[t]{6}{*}{ 1S } & Ideal & 1 & $<5$ \\
\hline & & Desirable & 2 & 5 to 9 \\
\hline & & Tolerable & 3 & 9 to 13 \\
\hline & & Undesirable & 4 & 13 to 17 \\
\hline & & $\begin{array}{l}\text { Highly } \\
\text { undesirable }\end{array}$ & 5 & 17 to 25 \\
\hline & & Unacceptable & $\mathrm{n} / \mathrm{a}$ & $>25$ \\
\hline \multirow{6}{*}{$\begin{array}{l}\text { Standard } \\
\text { deviation (3) }\end{array}$} & \multirow[t]{6}{*}{ 1S } & Ideal & 1 & $<0.5$ \\
\hline & & Desirable & 2 & 0.5 to 2.5 \\
\hline & & Tolerable & 3 & 2.0 to 3.0 \\
\hline & & Undesirable & 4 & 3.0 to 5.5 \\
\hline & & $\begin{array}{l}\text { Highly } \\
\text { undesirable }\end{array}$ & 5 & 5.5 to 7.0 \\
\hline & & Unacceptable & $\mathrm{n} / \mathrm{a}$ & $>7.0$ \\
\hline
\end{tabular}

class of the quality characteristic of interest. For soft classes, this would involve a re-determination of the designer's preferences to meet the specifications for the new class. For hard classes, the constraints in the model can be easily updated once the new target value is specified. The second possible type of categorization change involves case changes, where it is necessary to re-categorize soft cases as hard cases or vice versa. Here, the model can be updated to accommodate the new model constraints or revised AOF, respectively, depending on the necessary categorization change. The most significant design change possible in the proposed process is the addition or subtraction of quality characteristics to or from the model. If it is necessary to add a new quality characteristic to the design problem, the entire approach shown in Fig. 3 can be completed for the additional quality characteristic. Oppositely, if a quality characteristic is removed from the design problem, its constraints or weights are simply removed from the optimization model, which is then re-optimized, as discussed previously. The independent nature of the steps in the proposed process provides flexibility in terms of the design approach, such that changes to the design problem are easily incorporated into the existing model. Using this approach, new information is combined with the existing knowledge about the design to revise the model, as opposed to having to redesign the entire optimization model.

\subsection{Sensitivity analysis}

A sensitivity analysis was completed for the numerical example considered in this paper in order to study the effect of varying the input variables on the results of the proposed optimization model. In this investigation, which is shown in Table 7 , the design factors $\left(x_{1}{ }^{*}, x_{2}{ }^{*}, x_{3}{ }^{*}\right)$ for the tablet manufacturing problem are varied in the leftmost columns of the table and the calculations of the effect on the process mean $\left(\widehat{\mu}_{i}(\mathbf{x})\right)$ and standard deviation $\left(\widehat{\sigma}_{i}(\mathbf{x})\right)$, as well as the objective function (AOF) are shown on the right side of the table. The results of this study show how changes in design factors affect the outputs of the system under investigation, and the minimum value of the objective function is highlighted on the right side of the table. The results of our proposed methodology are validated by this study because the minimum value of the objective function corresponds to the optimum operating conditions found using our proposed methodology, which are highlighted on the left side of Table 7.
Table 6 The optimal design factor settings for the tablet manufacturing problem

\begin{tabular}{lcccccccc}
\hline$x_{1}{ }^{*}$ & $x_{2}{ }^{*}$ & $x_{3}{ }^{*}$ & $\widehat{\mu}_{1}\left(\mathbf{x}^{*}\right)$ & $\widehat{\sigma}_{1}\left(\mathbf{x}^{*}\right)$ & $\widehat{\mu}_{2}\left(\mathbf{x}^{*}\right)$ & $\widehat{\sigma}_{2}\left(\mathbf{x}^{*}\right)$ & $\widehat{\mu}_{3}\left(\mathbf{x}^{*}\right)$ & $\widehat{\sigma}_{3}\left(\mathbf{x}^{*}\right)$ \\
\hline-0.844 & 1.070 & 1.257 & 252.65 & 0.014 & 68.482 & 0.038 & 13.734 & 5.475
\end{tabular}




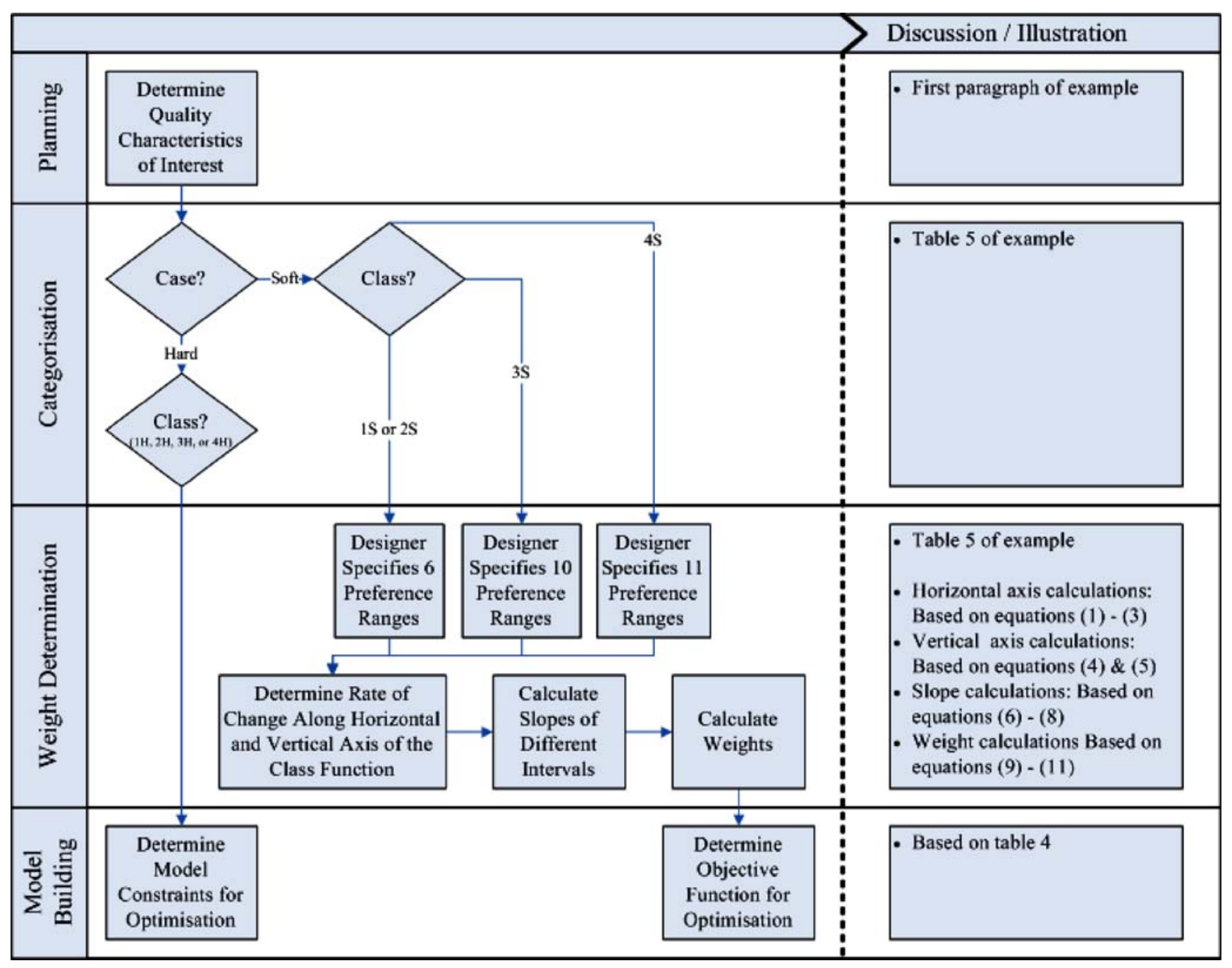

Fig. 3 Flowchart of the process followed in the tablet manufacturing problem

Table 7 Sensitivity analysis for the tablet manufacturing problem

\begin{tabular}{|c|c|c|c|c|c|c|c|c|c|}
\hline$x_{1}{ }^{*}$ & $x_{2}{ }^{*}$ & $x_{3}{ }^{*}$ & $\widehat{\mu}_{1}\left(\mathbf{x}^{*}\right)$ & $\widehat{\sigma}_{1}\left(\mathbf{x}^{*}\right)$ & $\widehat{\mu}_{2}\left(\mathbf{x}^{*}\right)$ & $\widehat{\sigma}_{2}\left(\mathbf{x}^{*}\right)$ & $\widehat{\mu}_{3}\left(\mathbf{x}^{*}\right)$ & $\widehat{\sigma}_{3}\left(\mathbf{x}^{*}\right)$ & $\mathrm{AOF}$ \\
\hline-0.840 & 1.043 & 1.306 & 256.10 & 0.016 & 69.465 & 0.105 & 13.933 & 5.832 & 8.7895 \\
\hline-0.676 & 1.138 & 1.349 & 265.60 & 0.685 & 68.385 & 0.005 & 14.32 & 5.414 & 8.0722 \\
\hline-0.909 & 1.037 & 1.223 & 247.90 & 0.019 & 68.731 & 0.325 & 13.787 & 5.809 & 9.1810 \\
\hline-0.821 & 1.067 & 1.285 & 255.30 & 0.149 & 68.896 & 0.112 & 13.93 & 5.667 & 7.4873 \\
\hline-0.648 & 1.131 & 1.377 & 268.46 & 1.074 & 69.011 & 0.333 & 14.798 & 5.898 & 10.600 \\
\hline-0.668 & 1.128 & 1.363 & 266.73 & 0.888 & 68.840 & 0.214 & 14.595 & 5.740 & 8.9154 \\
\hline-0.844 & 1.070 & 1.257 & 252.62 & 0.014 & 68.482 & 0.038 & 13.734 & 5.475 & 6.2544 \\
\hline-0.804 & 1.073 & 1.301 & 257.15 & 0.129 & 68.938 & 0.027 & 13.930 & 5.602 & 6.7051 \\
\hline-0.658 & 1.141 & 1.363 & 267.33 & 0.833 & 68.528 & 0.088 & 14.484 & 5.53 & 6.9503 \\
\hline-0.829 & 1.081 & 1.251 & 252.73 & 0.117 & 68.294 & 0.066 & 13.789 & 5.443 & 9.0307 \\
\hline-0.602 & 1.161 & 1.401 & 272.52 & 1.167 & 68.582 & 0.184 & 14.796 & 5.634 & 8.2775 \\
\hline-0.785 & 1.084 & 1.303 & 257.99 & 0.232 & 68.810 & 0.041 & 13.997 & 5.577 & 6.6495 \\
\hline-0.806 & 1.075 & 1.280 & 255.41 & 0.303 & 68.806 & 0.203 & 14.060 & 5.721 & 8.3045 \\
\hline
\end{tabular}




\section{Conclusion}

In this paper, we proposed an extension to the multiresponse robust design (RD) problem considering the use of physical programming. We described our proposed methodology in detail, demonstrated it through the use of a numerical example, and validated our findings with a sensitivity study. The model proposed here is unique because it incorporates a methodology for clearly defining priorities in multiresponse problems. Here, physical programming was used in the context of RD as a precise tool for capturing designer's preferences with a reasonable degree of accuracy and transforming these into specific weighted objectives. This approach, along with incorporating goal programming techniques into the traditional multiresponse RD problem, ensures the flexibility necessary in the development phase.

Our proposed model considers both multiple, conflicting objectives and design flexibility, in that changes to the design problem, which inevitably occur during the design process, are easily accommodated by our approach. By extending the current knowledge of physical programming and linking the concept of experimental design to RD, this paper develops the logistics of experiment-based RD with the consideration of multiple quality characteristics. As knowledge about the design evolves throughout the design timeline, the optimization model is easily adjusted in order to determine new optimum operating conditions. It is the independence of the model components in this approach that allows the model to be updated easily when given new information. The model is updated in such a way that revised information can be combined with prior information about the problem so that the model can be easily re-solved with minimal effort. Therefore, the proposed methodology supports human judgment involved in the decision-making process encountered in all design problems and allows products and processes to be designed more efficiently to meet customers' needs. The source code concerning the optimization software used in this paper, PhyOpt, can be forwarded upon a request made to the authors.

\section{References}

1. Taguchi GW (1986) Introduction to quality engineering. Krauss International Publications, White Plains, New York

2. Taguchi GW (1987) Systems of experimental design: engineering methods to optimize quality and minimize cost. Quality Resources, White Plains, New York

3. Taguchi GW, Wu YI (1985) Introduction to off-line quality control. American Supplier Institute, Dearborn, Michigan
4. Phadke MS (1989) Quality engineering using robust design. Prentice Hall, Englewood Cliffs, New Jersey

5. Kackar RN (1985) Off-line quality control, parameter design, and the Taguchi method. J Qual Tech 17(4):176-188

6. Kackar RN (1986) Taguchi's quality philosophy: analysis and commentary. Qual Prog 19(12):21-29

7. Leon RV, Shoemaker AC, Kackar RN (1987) Performance measures independent of adjustment: an explanation and extension of Taguchi's signal-to-noise ratios. Technometrics 29(3):253-285

8. Box GEP (1988) Signal-to-noise ratios, performance criteria, and transformations. Technometrics 30(1):1-17

9. Box GEP, Bisgaard S, Fung C (1988) An explanation and critique of Taguchi's contributions to quality engineering. Int J Relia Mgt 4(2):123-131

10. Pignatiello JJ Jr, Ramberg JS (1991) Top ten triumphs and tragedies of Genichi Taguchi. Qual Eng 4(2):211-225

11. Nair VN (1992) Taguchi's parameter design: a panel discussion. Technometrics 34(2):127-161

12. Tsui KL (1992) An overview of Taguchi method and newly developed statistical methods for robust design. IIE Trans 24 (5):44-57

13. Myers RH (1999) Response surface methodology — current status and future directions. J Qual Tech 31(1):30-44

14. Vining GC, Myers RH (1990) Combining Taguchi and response surface philosophies: a dual response approach. J Qual Tech 22 (1):38-45

15. del Castillo E, Montgomery DC (1993) A nonlinear programming solution to the dual response problem. J Qual Tech 25(3):199-204

16. Copeland KAF, Nelson PR (1996) Dual response optimization via direct function minimization. J Qual Tech 28(3):331-336

17. Cho BR (1994) Optimization issues in quality engineering. $\mathrm{PhD}$ thesis, University of Oklahoma, Oklahoma

18. Lin DKJ, Tu W (1995) Dual response surface optimization. J Qual Tech 27(1):34-39

19. Kim YJ, Cho BR (2000) Economic consideration on parameter design. Qual Reliab Eng Int 16:501-514

20. Parkinson DB (2000) Robust design employing a genetic algorithm. Qual Reliab Eng Int 16(3):201-208

21. Brenneman WA, Myers WR (2003) Robust parameter design with categorical noise variables. J Qual Tech 35(4):335-341

22. Köksoy O, Doganaksoy N (2003) Joint optimization of mean and standard deviation using response surface methods. J Qual Tech 35(3):239-252

23. Miro-Quesada G, del Castillo E (2004) Two approaches for improving the dual response method in robust parameter design. J Qual Tech 36(2):154-168

24. Shin SM, Cho BR (2005) Bias-specified robust design optimization and its analytical solutions. Comp Ind Eng 48(1):129-140

25. Harrington EC (1965) The desirability function. Ind Qual Cont 21 (10):494-498

26. Derringer G, Suich R (1980) Simultaneous optimization of several response variables. J Qual Tech 12(4):214-219

27. Khuri AI, Conlon M (1981) Simultaneous optimization of multiple responses represented by polynomial regression functions. Technometrics 23(4):363-375

28. Pignatiello JJ (1993) Strategies for robust multi-response quality engineering. IIE Trans 25(3):5-15

29. Messac A (1996) Physical programming: effective optimization for computational design. AIAA J 34(1):149-158

30. Messac A (2000) From the dubious construction of objective functions to the application of physical programming. AIAA J 38 (1):155-163

31. Messac A, Gupta SM, Akbulut B (1996) Linear physical programming: a new approach to multiple objective optimization. Trans Oper Res 8(1):39-59 
32. Chen W, Sahai A, Messac A, Sundararaj GJ (2000) Exploration of the effectiveness of physical programming in robust design. J Mech Des 122(2):155-163

33. Messac A, Ismail-Yahaya A (2001) Multiobjective robust design using physical programming. Struc Multidiscip Opt 23(5):357371

34. Logothetis N, Haigh A (1988) Characterizing and optimizing multi-response processes by Taguchi method. Qual Relia Eng Int 4(2):159-169

35. Elsayed EA, Chen A (1993) Optimal levels of process parameters for products with multiple characteristics. Int J Prod Res 31 (5): $1117-1132$

36. Ames AE, Mattucci N, MacDonald S, Szonyi G, Hawkins DM (1997) Quality loss functions for optimization across multiple response surfaces. J Qual Tech 29(3):339-346

37. Derringer GC (1994) A balancing act: optimizing a product's properties. Qual Prog 27(6):51-58

38. Kapur KC, Cho BR (1996) Economic design of the specification region for multiple quality characteristics. IIE Trans 28(3):237-248

39. Su C-T, Tong L-I (1997) Multi-response robust design by principal component analysis. Total Qual Mgt 8(6):409-416

40. Tong L-I, Su C-T (1997) Optimizing multi-response problems in the Taguchi method by fuzzy multiple attribute decision making. Qual Relia Eng Int 13(1):25-34

41. Tong L-I, Su C-T, Wang C-H (1997) The optimization of multiresponse problems in the Taguchi method. Int J Qual and Relia Mgt 14(4):367-380

42. Chen L-H (1997) Designing robust products with multiple quality characteristics. Comp Oper Res 24(10):937-944
43. Kim K, Lin DKJ (1998) Dual response surface optimization: a fuzzy modeling approach. J Qual Tech 30(1):1-10

44. Vining GG (1998) A compromise approach to multiresponse optimization. J Qual Tech 30(3):309-313

45. Tsui KL (1999) Robust design optimization for multiple characteristic problems. Int J Prod Res 37(2):433-445

46. Chiao C-H, Hamada M (2001) Analyzing experiments with correlated multiple responses. J Qual Tech 33(4):451-465

47. Kim YJ, Cho BR (2002) Development of priority-based robust design. Qual Eng 14(3):355-363

48. Tang LC, Xu K (2002) A unified approach for dual response surface optimization. J Qual Tech 34(4):437-447

49. Lin CL, Lin JL, Ko TC (2002) Optimization of the EDM process based on the orthogonal array with fuzzy logic and grey relational analysis method. Int J Adv Manuf Tech 19(4):271-277

50. Lu D, Antony J (2002) Optimization of multiple responses using a fuzzy-rule based inference system. Int J Prod Res 40(7):1613-1625

51. Romano D, Varetto M, Vicario G (2004) Multiresponse robust design: a general framework based on combined array. J Qual Tech 36(1):27-37

52. Wu F-C, Chyu C-C (2004) Optimization of robust design for multiple quality characteristics. Int J Prod Res 42(2):337-354

53. Hillier FS, Lieberman GJ (2001) Introduction to operations research. McGraw-Hill, New York

54. Bourquin J, Schmidli H, van Hoogevest P, Leuenberger H (1998) Pitfalls of artificial neural networks (ANN) modelling technique for data sets containing outlier measurements using a study on mixture properties of a direct compressed dosage form. Eur J Pharm Sci 7(1):17-28 